\title{
Episodic retrieval and feature facilitation in intertrial priming of visual search
}

\author{
Árni Gunnar Ásgeirsson • Árni Kristjánsson
}

Published online: 14 April 2011

(C) Psychonomic Society, Inc. 2011

\begin{abstract}
Huang, Holcombe, and Pashler (Memory \& Cognition, 32, 12-20, 2004) found that priming from repetition of different features of a target in a visual search task resulted in significant response time (RT) reductions when both target brightness and size were repeated. But when only one feature was repeated and the other changed, RTs were longer than when neither feature was repeated. From this, they argued that priming in visual search reflected episodic retrieval of memory traces, rather than facilitation of repeated features. We tested different variations of the search task introduced by Huang et al., with the aim of uncovering when priming is episodic and when feature based. We found that varying the signal strength of target against distractors had a strong effect on the priming pattern. In difficult search with low signal-to-noise ratios of target against distractors, the priming patterns were episodic. When feature contrasts between target and distractors were increased, priming of different features was independent and additive. Our results suggest that, during inefficient search, priming can be episodic but that, for more efficient search, priming from different features occurs independently. The results support two-stage (or multistage) accounts of priming in visual search.
\end{abstract}

Keywords Attention · Visual search · Priming

\footnotetext{
Á. G. Ásgeirsson $(\varangle) \cdot$ Á. Kristjánsson

School of Health Sciences, University of Iceland, Reykjavik, Iceland

e-mail: arni.asgeirsson@psy.ku.dk

Á. G. Ásgeirsson

Center for Visual Cognition, Department of Psychology,

University of Copenhagen,

Copenhagen, Denmark

A popular model of attentional selection is embodied in visual search tasks. A typical finding is that items that stand out against distractor items in a visual search array on features such as color, shape, or orientation will effortlessly "pop out" from among distractors (Julesz, 1984; Treisman \& Gelade, 1980; Wolfe, Cave, \& Franzel, 1989). An important qualification to this was, however, noted by Maljkovic and Nakayama (1994), who found that even such highly salient targets were found more quickly (and more accurately; see also Sigurdardottir, Kristjánsson, \& Driver, 2008) if the target-defining feature was repeated from one trial to the next, as compared with when target identity changed. The search was apparently not as "effortless" and automatic as previously thought.

Such repetition priming in visual search has attracted a lot of interest in recent years. Priming has been shown to have a surprisingly large effect on search times in various tasks (Geyer, Müller, \& Krummenacher, 2006; Hillstrom, 2000; Lamy, Bar-Anan, \& Egeth, 2008; Olivers \& Meeter, 2006) and can, in some cases, account for effects attributed to topdown guidance (see, e.g., Becker, 2008a; Kristjánsson, Wang, \& Nakayama, 2002; Wang, Kristjánsson, \& Nakayama, 2005; Wolfe, Butcher, Lee, \& Hyle, 2003; but see Lamy, Carmel, Egeth, \& Leber, 2006; Leonard \& Egeth, 2008). Many have argued that priming plays an important role in determining moment-to-moment attentional selection (Kristjánsson, 2006a; Lamy et al., 2006; Nakayama, Maljkovic, \& Kristjánsson, 2004; Wolfe et al., 2003).

Because of this importance of priming for visual perception, understanding its characteristics and the mechanisms behind it becomes all the more important. Two accounts of priming of visual search include feature facilitation (Becker, 2008a, 2008b; Kristjánsson, 2006a, 2008; Maljkovic \& Nakayama, 1994; Nakayama et al., 2004) and episodic retrieval (Hillstrom, 2000; Huang et al., 2004; Huang \& 
Pashler, 2005). Maljkovic and Nakayama (1994) tested performance in a single-feature visual search where the observers indicated whether there was a notch at the right or the left of a single-target diamond (either red or green) among two distractors of the other color, finding that repetition of target color speeded the search, as compared with when it changed. They argued that this reflected facilitation of attention shifts through altered saliency of the repeated feature. As a red target repeats, attention is drawn to red items in the visual field, and the distractor color (green) is inhibited (see, e.g., Becker \& Horstmann, 2009; Goolsby \& Suzuki, 2001; for related accounts, see Sigurdardottir et al., 2008; for a review, see Kristjánsson \& Campana, 2010).

A notable challenge to this feature facilitation view was reported by Huang et al. (2004). Their observers searched for an odd-sized bar among 19 distractor bars (target and distractors were either black or white, determined randomly) and reported its orientation $\left(+45^{\circ}\right.$ or $\left.-45^{\circ}\right)$. If the target was small, the distractors were large, and vice versa. Their most notable finding was that when the same-sized target was repeated, search was faster if target brightness was also repeated, but when target size was different from that on the previous trial, repetition of target brightness harmed performance. Priming from repetition of size was thus not independent of whether the targets' brightness was repeated or not. Huang et al. argued that the most parsimonious account for these results was that priming reflects facilitated processing of whole objects rather than single features - in particular, an episodic memory representation of the previous trial. They argued that when single features are repeated, the pattern is consistent with feature facilitation but that interaction effects emerge only when different features are repeated independently. Hillstrom (2000) also argued against feature facilitation accounts, proposing that priming does not affect the saliency of repeated features but reflects episodic memory traces of the foregoing trial.

Priming, according to this, affects a relatively late stage of the perceptual process. This was at odds with Maljkovic and Nakayama's (1994) claim that the attended feature was selectively facilitated (see also Kristjánsson, 2008; Nakayama et al., 2004), reflecting facilitation at earlier levels of processing. Feature facilitation accounts predict additive effects from repetition of different features, rather than an interaction between repetitions of the two features, since the repetition facilitation is feature specific, independently of the context.

But these results of Huang et al. (2004) disagree with more recent results of Kristjánsson (2006b, 2009), who found that repetition of different features of a Gabor target (color, orientation, spatial frequency, and motion) resulted in independent and additive priming of the different features. There was a priming benefit from repeating only one of the features even if the other changed. There was no interaction between repetition effects of the two. Kristjánsson (2006b) speculated that the episodic priming effects observed by Huang et al. might reflect priming at a higher level of processing than the independent priming he observed and that this might then reflect that priming effects occur at multiple stages of perceptual processing.

It is important to note that feature facilitation and episodic retrieval need not be mutually exclusive, and dual-stage (Lamy, Yashar, \& Ruderman, 2010) and multiplelevel (Kristjánsson \& Campana, 2010) accounts of priming have been proposed (see Kristjánsson, Ingvarsdóttir, \& Teitsdóttir, 2008, for direct evidence for such dual-stage priming).

\section{Present aims}

The aim here was to elucidate under what conditions we might expect priming patterns indicative of episodic retrieval, where both features must be repeated for repetition facilitation and when there is independent priming of different features. We introduced a number of variations upon the paradigm introduced by Huang et al. (2004). In Experiment 1, we attempted to replicate their critical experiment. In the experiments that followed, we attempted to uncover the task characteristics leading to interactive priming effects. Experiment 2 was designed to address the question of whether between-trial role-reversals of target and distractors in Huang et al.and the present Experiment 1 cause interactions between repetitions of size and brightness. Role-reversals have notable effects in visual search tasks, independently of target and distractorset repetition effects (Kristjánsson \& Driver, 2008). Furthermore, repetition of distractor sets has a dramatic effect on search performance (Geyer et al., 2006; Kristjánsson \& Driver, 2008; Lamy et al., 2008) —in some cases, even stronger than the effects of target repetition (Geyer et al., 2006). In Experiment 2, we therefore used targets and distractors similar to those in Huang et al., except that distractor size was always constant, while the targets were either smaller or larger than the distractors. In Experiments 1 and 2, we also varied set-size between trials in order to test task difficulty.

In Experiment 3, we further investigated the role of target versus distractor discriminability in creating the interaction between repetitions of size and brightness observed by Huang et al. (2004) by increasing the size difference between the two different possible sizes, thus reducing the difficulty of the search. In Experiment 4, we further attempted to increase the signal strength of target against distractors by reversing the roles of size and brightness. The target was defined by brightness (black/white), while size was the irrelevant dimension. 
To preview the results, we found that for search where the salience of target against distractors was relatively low, there were episodic interactions between repetition effects from different features, whereas when task parameters changed so that the salience of target against distractors increased, priming of different features was independent and additive. This supports the view that priming in visual search reflects modulation from repetition at different levels of processing, depending on the task.

\section{Experiment 1: Replicating Huang et al. (2004)}

In Experiment 1, we set out to replicate the results of Huang et al. (Experiment 1), with the only difference being that set-size was varied, to measure the difficulty of the search.

\section{Method}

Participants Eight students at the University of Iceland with normal or corrected-to-normal vision participated. Seven were unaware of the purpose of the experiment, while the 8th was the first author (Á.G.Á.).

Stimuli and apparatus A 400-MHz Macintosh computer was used to generate the stimuli, presented on a 14-in. 65-Hz CRT monitor, and to collect responses via keypress. An area of approximately $24^{\circ} \times 24^{\circ}$ of visual angle on the screen constituted the search array. Viewing distance was $40 \mathrm{~cm}$. The Vision Shell ${ }^{\circledR}$ toolbox (Comtois, 2003) for C was used to program the stimuli and to collect responses. Set-size varied randomly between 16,32 , or 48 . The observers responded whether the target, an odd-sized bar, present on every trial, was oriented to the left or the right of vertical $\left( \pm 45^{\circ}\right.$, varying randomly between trials). The target varied randomly in length $\left(1^{\circ}\right.$ or $1.6^{\circ}$; width $\left.=0.4^{\circ}\right)$ and randomly in brightness (black, $<1 \mathrm{cdm}^{-2}$; white, $80 \mathrm{cdm}^{-2}$ ). The distractors were always of opposite length to the target (see Fig. 1a). The target was thus either a $1^{\circ}$ bar among $1.6^{\circ}$ distractor bars or vice versa, so that if the target identity changed between trials, the target identity became the distractor identity. The search items were presented on a mid-gray $\left(22 \mathrm{cdm}^{-2}\right)$ background.

Procedure and design The observers were told to respond as quickly and accurately as possible to the orientation of the odd-sized singleton target. They responded with the index and ring fingers of their right hand by pressing $4\left(-45^{\circ}\right.$ tilt) or 6 $\left(+45^{\circ}\right.$ tilt) on the keyboard number pad. A high-pitched tone designated the start of a trial; a medium-pitched tone denoted a correct response; and a low-pitched tone an incorrect response. Trials with incorrect responses or response times (RTs) \pm 3 standard deviations from each mean RT were discarded. Each observer participated in 300 trials completed in a single block, preceded by 30 practice trials.

\section{Results and discussion}

In Experiment 1, we replicated Huang et al.'s (2004) finding of an interaction between repetitions of two different features of the singleton target. This result argues against pure feature facilitation accounts of priming, because the effects of repetitions of brightness and size are not independent, since the whole target object needs to
Fig. 1 Design and results in Experiment 1. a typical search array in Experiment 1, showing a large black target with smaller black and white distractors. b Mean response times as a function of whether brightness and size were repeated or changed from the preceding trial. The error bars show the standard deviations of the means a

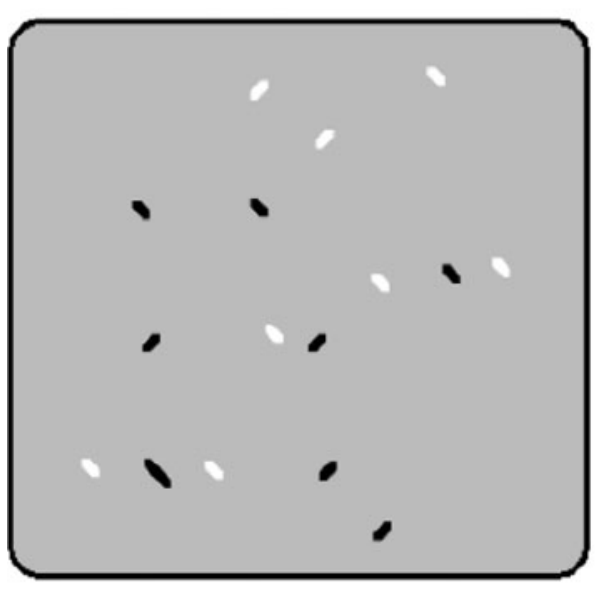

b

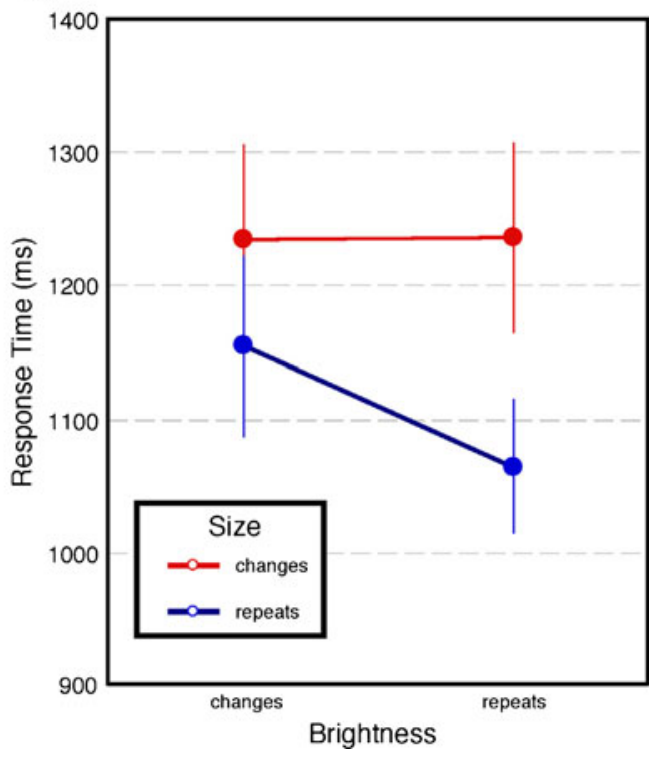


be repeated for priming to occur (see also Kristjánsson et al., 2008). We also observed a large effect of set-size. As more items were added to the display, the longer the search times became.

RTs as a function of whether target size, brightness, or both were repeated or not are shown in Fig. 1 b. A $2 \times 2$ repeated measures ANOVA revealed a main effect of size repetition $(126 \mathrm{~ms}), F(1,7)=11.437, p=.012$, but the main effect of brightness repetition was only marginally significant, $F(1,7)=4.873, p=.063$. As in Huang et al. (2004), Experiment 1, the interaction between size repetition and brightness repetition was significant, $F(1,7)=$ 8.466, $p=.023$. These results are in agreement with Experiment 1 in Huang et al.and support the claim that repetition of both target features is necessary for repetition facilitation in this paradigm.

A set-size $\times$ size repetition $\times$ brightness repetition ANOVA performed to explore possible effects of set-size on RTs revealed similar main effects of both size and brightness repetition and an interaction between those variables. But there was also a highly significant main effect of set-size, $F(2,14)=26.449, p<.001$; the RT versus set-size slope was $8 \mathrm{~ms}$ per item. There was no interaction between setsize and feature repetition.

Splitting the results into short and long $R T S$ The variance of RTs in this experiment was rather large. We therefore split the data for each observer into two groups at the median. For the lower-than-median RTs, there was a significant effect of repeating size, $F(1,7)=77.464, p<.001$, and the effect of brightness repetition was close to significant, $F(1,7)=3.783, p=.093$, but most important, there was no interaction, $F(1,7)=0.212, p=.659$. For the higher-thanmedian RTs, there was a main repetition effect of size in the direction opposite to that expected, $F(1,7)=17.488$, $p=.004$. There was no effect of brightness repetition, $F(1,7)=0.057, p=.818$, and no interaction, $F(1,7)=$ $0.013, p=.912$.

In sum, only when all the RTs were included was there an interaction indicative of episodic priming, suggesting that task difficulty determined the priming pattern observed in each case. In the experiments that follow, we pursued this further.

Splitting the results by target and distractor size We also analyzed the data on the basis of groups of large versus small target stimuli. There is reason to believe that search for a large bar among small bars is quite different from search for a small bar among large ones, since there is a large difference in the crowdedness of the search array. A comparison between the two conditions supports this. Finding a large target among small distractors took 1,077 $\mathrm{ms}$, on average, while the average RT was $1,319 \mathrm{~ms}$ when observers searched for a small target among large distractors, $F(1,7)=54.898, p<.001$.

When the target was large (and the distractors small), there was a significant effect of size repetition, $F(1,7)=$ $26.764, p=.001$, while neither brightness repetition effects nor the interaction was significant $(p s=.121$ and .122 , respectively). However, when the target was a small bar among large ones, the effects were reversed. Now the size repetition effect was not significant, $F(1,7)=2.932$, $p=.131$, while the brightness repetition effect, $F(1,7)=$ $22.921, p=.002$, and the interaction, $F(1,7)=5.797$, $p=.047$, were significant. This is consistent with the results of the median split on the RTs, which indicated that the interaction observed by Huang et al. (2004) emerges as the search becomes difficult.

Table 1 shows the error rates from all the experiments reported here. A $2 \times 2$ repeated measures ANOVA did not reveal any effects of feature repetition on error rates in Experiment 1, indicating no speed-accuracy trade-offs as a function of different repetition combinations.

To summarize, in Experiment 1, we showed that the basic findings of Huang et al. (2004) are replicable. But when the results for each observer were split along their median RT value, an interesting pattern emerged where, for low RTs, priming from different features was independent and additive but the pattern for high RTs was not as clear. Only when all the data were included in the analysis was there an interaction between repetition effects of different features. This suggests that search difficulty may play an
Table 1 Error rates in all four experiments as a function of condition

\begin{tabular}{|c|c|c|c|c|c|c|c|c|}
\hline \multirow[t]{2}{*}{ Exp. } & \multicolumn{2}{|c|}{ Both Change } & \multicolumn{2}{|c|}{ Size Repeated } & \multicolumn{2}{|c|}{ Brightness Repeated } & \multicolumn{2}{|c|}{ Both Repeated } \\
\hline & Mean $\%$ & SEM & Mean \% & SEM & Mean \% & SEM & Mean \% & SEM \\
\hline 1 & 3.7 & 1.2 & 3.5 & 1.0 & 2.8 & 0.5 & 2.3 & 1.0 \\
\hline 2 & 4.8 & 0.9 & 2.5 & 0.4 & 1.9 & 0.8 & 2.0 & 1.0 \\
\hline 3 & 3.2 & 1.4 & 3.8 & 1.0 & 3.3 & 1.0 & 2.1 & 1.0 \\
\hline 4 & 3.0 & 0.5 & 2.9 & 1.0 & 3.0 & 0.8 & 2.4 & 0.7 \\
\hline
\end{tabular}


important role in producing the priming pattern. Consistent with this, the interaction is seen only for search for a small target among large distractors, not vice versa. The results also show that the search is inefficient with large set-size effects. In the experiments that follow, we therefore explored the role of target versus distractor discriminability in producing the interaction between repetition effects of different features.

\section{Experiment 2: Effects of role reversals between target and distractors}

In Experiment 1 and in Huang et al. (2004), repetition of target and distractor features was confounded. When target size changed, the distractors took on the size of the previous target, and vice versa. Distractor set priming plays a large role in priming in visual search (Kristjánsson et al., 2002; Lamy et al., 2008; Saevarsson, Jóelsdóttir, Hjaltason, \& Kristjánsson, 2008) - a role that may be as large as the target priming effects or even larger (Geyer et al., 2006). Also, role-reversals between target and distractors have strong effects on search performance, an effect that is independent of the effects of target and distractor priming (Kristjánsson \& Driver, 2008). In Experiment 2, we examined effects from role-reversals by keeping the size of the distractors constant, while the target was either larger or smaller than the distractors. Note that this manipulation also abolished search array differences when participants searched for large versus small bars. Crowding effects should be comparable regardless of whether the target is large or small when set-size is constant.

Method

Participants Seven naïve observers and the first author participated. All reported normal or corrected-to-normal vision.

Stimuli and apparatus The targets were black or white tilted bars (as in Experiment 1), varying randomly between being larger or smaller than the distractors (see Fig. 2a). Distractor size was constant throughout $\left(1.9^{\circ}\right)$. We matched the salience of small and large bars by pilot-testing 5 observers on a number of different target sizes. Bars of $1^{\circ}$ and $3.2^{\circ}$ were the closest approximation to equal salience, in that the RTs were comparable. Set-size varied randomly between 10,20, and 30 items. The apparatus, procedure, and design were similar to those in Experiment 1. Each observer participated in 500 trials.

\section{Results and discussion}

With Experiment 2, we addressed the importance of rolereversals between target and distractors in the original experiment of Huang et al. (2004). The results (in Fig. 2b) show that when the roles of target and distractors do not reverse - the distractors are always of constant size - there
Fig. 2 Design and results in Experiment 2. a typical search arrays in Experiment 2, showing a small black target among 9 medium-sized distractors (above) and a large white target among 29 medium-sized distractors (below). b Mean response times as a function of whether brightness and sizewererepeated or not. The error bars represent $\pm 1 S E M$ a
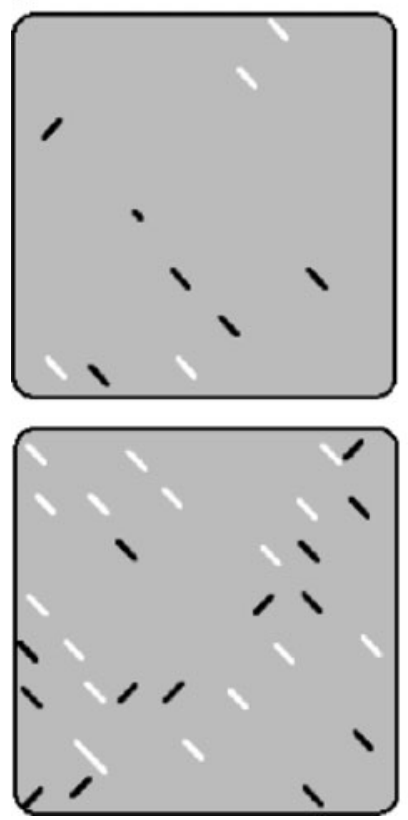

b

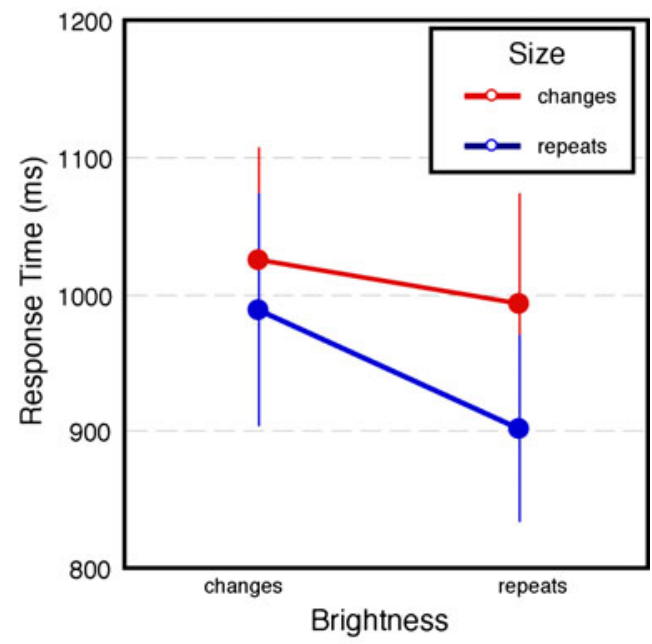


is no interaction between repetitions of brightness and size. A $2 \times 2$ repeated measures ANOVA showed a main effect of brightness repetition, $F(1,7)=7.933, p=.026$, and a marginally significant effect of size repetition, $F(1,7)=$ $5.076, p=.059$. There was no interaction at all between repetition effects from the two features, $F(1,7)=0.829$, $p=.393$.

A $3 \times 2 \times 2$ repeated measures ANOVA, with set-size as the added factor, confirmed a main effect of brightness repetition, $F(1,7)=16.604, p=.005$, but the size repetition effect was not quite significant, $F(1,7)=3.474, p=.105$. This ANOVA also showed a large main effect of set-size, $F(1.059,7.414)^{1}=24.637, p=.001$, corresponding to a search slope of $20.6 \mathrm{~ms}$ per item added to the display. No interactions were found. The effects of repeating size and brightness were independent.

As in Experiment 1, we split the results for each observer at the median, with very different patterns depending on whether the search times were short or long. For the lower-than-median RTs, there was a significant main effect of size repetition, $F(1,7)=22.866, p=.002$, and the effect of brightness repetition was close to significant, $F(1,7)=4.423, p=.074$. There was no hint of an interaction, $F(1,7)=1.509, p=.259$. For the higherthan-median RTs, neither the main effects nor the interaction was significant. Again, this indicates that task difficulty plays a crucial role in determining the priming patterns.

A $2 \times 2$ repeated measures ANOVA on the error rates revealed an interaction between brightness and size repetition, $F(1,7)=7.803, p=.027$. When one or both features were repeated, participants made few errors, while alternating both features increased the error rates (Table 1), suggesting that the search is most difficult when both features change, consistent with the RT results.

In Experiment 2, there was no interaction between the repetitions of brightness and size. Target and distractor role-reversals seemingly strongly affected the repetitionpriming pattern, since the only notable change in the design from Experiment 1 was that distractor size remained constant and target size varied. We speculate that role reversals increase the difficulty of determining target identity. Once again, this raises the question of whether target discriminability against distractors determines the priming pattern. This is even more likely when we consider the results from the median split of the RTs. In Experiment 3, we further pursued this idea by increasing the signal strength of the target against distractors, as compared with Experiment 1.

\footnotetext{
${ }^{1}$ Greenhouse-Geisser corrected degrees of freedom due to significant deviations from sphericity.
}

\section{Experiment 3: Increasing target signal strength}

Experiment 2 showed that when target/distractor confusion is decreased by eliminating role-reversals, there are no interactions between repetitions of different features. In Experiment 3, we directly manipulated target/distractor discriminability. If the episodic interaction is caused by confusion over target identity, increasing the signal strength of target against distractors might then lessen such confusion and the interaction between repetitions of different features.

A difference in saliency for the reported and irrelevant features may influence the priming pattern - for example, if in the difficult task, priming operates on assembled objects (as suggested by Kristjánsson et al., 2008). To test this, we increased the size difference between targets and distractors, making the size difference (the target-defining dimension) more salient among the high-contrast brightness variations of the distractors (irrelevant dimension). Our question was whether an easier task with otherwise comparable stimuli would result in an interaction between repetitions of different features.

\section{Method}

Participants Eight naïve observers and the first author participated. All had normal or corrected-to-normal vision.

Stimuli and apparatus Brightness contrast was similar to that in Experiment 1 and 2 (black vs. white), but the size difference between targets and distractors increased to $1^{\circ}$ versus $2.6^{\circ}$ (as compared with $1^{\circ}$ vs. $1.6^{\circ}$ in Experiment 1 ). Set size was fixed at 20. Apparatus, procedure, and design were otherwise identical to those in the previous experiments. Each observer participated in 300 trials.

\section{Results and discussion}

We have speculated that search difficulty played an important role in the interaction supporting episodic retrieval accounts found in Experiment 1. Here, we increased the size difference between targets and distractors to decrease search difficulty. The results show that by increasing the saliency of target against distractors, there is no episodic interaction, and the priming pattern reflects facilitation of repeated individual features.

As was expected, increases in size contrast lowered RTs (Fig. 3). Mean RTs ranged from $699 \mathrm{~ms}$ when neither feature was repeated to $657 \mathrm{~ms}$ when both features were repeated. A $2 \times 2$ repeated measures ANOVA showed a main effect of size repetition, $F(1,8)=33.894, p<.001$, and brightness repetition, $F(1,8)=11.937, p=.009$. There 


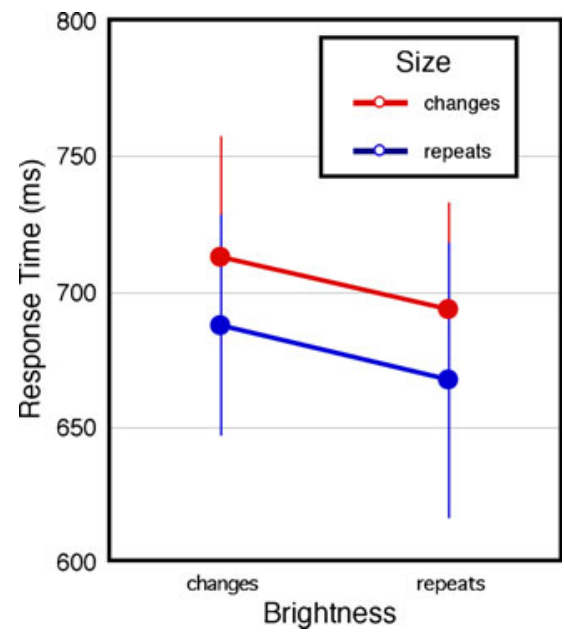

Fig. 3 Results from Experiment 3 as a function of whether brightness, size, or both were repeated or not. The error bars represent $\pm 1 S E M$

was no interaction between repetitions of the two variables, $F(1,8)=0.18, p=.682$. In other words, an interaction between repetitions of the two features is not seen when size differences between target and distractors are increased. A $2 \times 2$ repeated measures ANOVA revealed no speedaccuracy trade-offs under the different conditions (Table 1).

The priming from feature repetition was additive in Experiment 3. RTs were shortest when both features were repeated and were longest when both features changed. As search became easier, repetition priming effects from different features did not interact. Along with Experiment 2 , this result indicates that target/distractor discriminability plays a key role in whether repetition effects from different features interact or not, perhaps because, with increased difficulty, priming effects occur at later stages of perceptual processing.

\section{Experiment 4: Changing target dimension from size to brightness}

In Experiment 4, we examined the effect of changing the roles of the individual features on priming. Brightness was the target-defining variable, while size varied randomly. In pilot studies, we noted that this manipulation resulted in easier search, presumably reflecting that search is easier for the brightness singleton than for the size singleton (see, e.g., Hillstrom, 2000; Theeuwes, 1991). If increased difference between target and distractors causes feature-based priming, this should then also hold for Experiment 4.

Method

Participants Six naïve observers participated along with the first author.
Stimuli and apparatus Observers searched for a target of odd brightness relative to distractors (black among white or white among black). The irrelevant variable, size, varied randomly between large $\left(1.6^{\circ}\right)$ and small $\left(1.0^{\circ}\right)$ for each item in the display. Set-size on each trial varied randomly between 10,20, and 30. Otherwise the methods were similar to that in the previous experiments. Each observer completed 30 practice trials before performing two blocks of 200 trials.

\section{Results}

Experiment 4 showed that when the task in Experiment 1, where we observed an episodic interaction, was modified so that brightness became the target-defining feature instead of size, the task became easier. And importantly, there was no interaction between repetition effects from different features. Search was slowest when neither of the target features was repeated and fastest when both features were repeated. Once again, this result shows how increasing the signal strength of target against distractors abolishes the interaction between repetitions of the different features found by Huang et al. (2004; see also Hillstrom 2000, Experiment 1, for converging results with a set-size of 3).

The results from Experiment 4 are shown in Fig. 4. A $2 \times 2$ repeated measures ANOVA showed a 53-ms main effect of brightness repetition, $F(1,6)=120.474, p<.0001$, but the size repetition effect was not quite significant $(8 \mathrm{~ms})$, $F(1,6)=4.487, p=.078$. There was no interaction, $F(1,6)=$ $0.228, p=.65$.

A $3 \times 2 \times 2$ ANOVA did not reveal any set-size effects $(p=.292)$ nor an interaction between set-size and repetition of either feature $(p s>357)$. A $2 \times 2$ ANOVA did not show

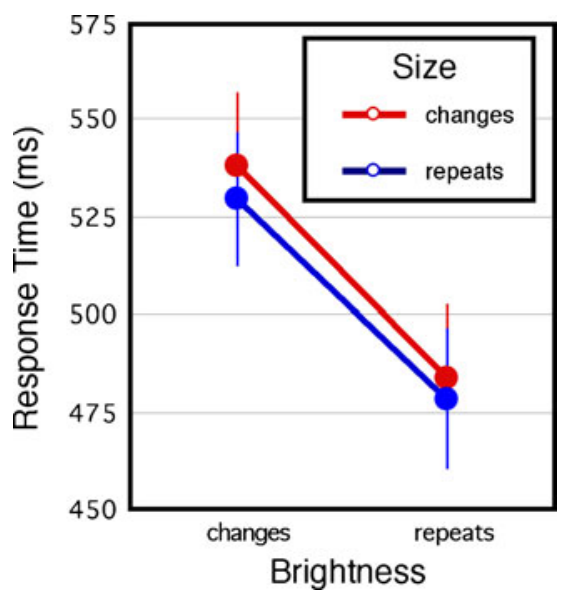

Fig. 4 Mean response times as a function of repetition and alternations in Experiment 4. Error bars represent \pm 1 SEM 
effects of feature repetition on error rates $(p \mathrm{~s}>.38$; see error rates in Table 1).

Experiment 4 suggests that episodic interactions do not occur in search where target and distractors are highly dissimilar. This is consistent with the postulate that as search becomes easier, priming patterns are feature based, whereas episodic interactions between repetitions of different features can be seen for harder search tasks.

The results support two stage accounts of priming (Lamy et al., 2010; see also Kristjánsson \& Campana, 2010). In difficult search, priming effects reflect modulations at a stage where objects are integrated, while priming of individual features is seen for easier search tasks (Kristjánsson et al., 2008).

\section{General discussion}

Episodic retrieval accounts have been proposed as alternatives to feature-weighting accounts of priming in visual search. Huang et al. (2004) showed that priming patterns in a difficult singleton search task can be explained well under episodic retrieval accounts, and this result has been replicated here. This is in contrast to other results on priming where different features led to independent priming effects (Kristjánsson 2006b, 2009). We have investigated the priming patterns in the general experimental design introduced by Huang et al. and some modifications of their task and draw the following conclusions.

1. Episodic priming is seen mainly in difficult searches. The interaction between repetitions of different features found by Huang et al. (2004) was found in a difficult task where the feature contrast of the target-defining variable was low.

2. By manipulating target-distractor discriminability, we show how increasing the signal strength and decreasing target and distractor confusion abolishes the interaction between repetition effects from different features.

3. Experiment 2 shows how episodic interactions disappear when role-reversals between target and distractors are eliminated, perhaps through increased discriminability of target against distractors.

4. In Experiments 3 and 4, where we (1) increased the size difference between target and distractors and (2) reversed the roles of brightness and size, greatly reducing the difficulty of the task, there was no evidence of an interaction between repetition of the defining and irrelevant dimensions, again suggesting that signal strength of target against distractors plays a vital role in the interaction.

5. We conclude that episodic interactions between effects of repeating different features reflect priming effects at late stages of perceptual processing, along the lines discussed by Kristjánsson et al. (2008) and Lamy et al. (2010; see also Kristjánsson \& Campana, 2010).

Overall, the interaction between repetition effects from different features observed by Huang et al. (2004) is problematic for pure feature facilitation accounts of priming. But this interaction seems mainly to occur in tasks in which the salience of the target against distractors is low. The interaction may emerge with search strategies different from those employed in efficient search.

Episodic retrieval accounts of priming are, therefore, not alternatives to feature facilitation accounts. At the same time, the presence of these interaction effects under certain conditions suggests that priming may operate at two levels of processing. Feature priming may reflect a relatively early stage, where objects are not fully integrated so that priming from the two different features proceeds independently. For difficult tasks such as those in Experiment 1 here and Huang et al. (2004), repetition benefits reflect processing at stages where objects are integrated and are seen only when both features are repeated.

Lamy et al. (2010) argued for a dual-stage account of priming, claiming that what we have hitherto referred to simply as priming are, in fact, two different phenomena (see also Kristjánsson \& Campana, 2010; Kristjánsson et al., 2008). With a dual-stage account, we infer the existence of an initial sampling stage (or early perceptual stage in Lamy et al., 2010) where recently sampled features are resampled more efficiently than others and a later stage that benefits from temporarily stored information about previously sampled (whole) objects or priorities on previous trials. The results here are completely consistent with this. Under a multistage account, we might also include a response stage, which may be influenced by repetition, as Huang et al. (2004) concluded. But we believe that the search task introduced by Huang et al. and used here is not optimal for answering such questions, since the response variable (orientation) is such a salient part of the stimuli and its repetition is $100 \%$ correlated with repetition of the response. Therefore, we cannot dissociate the effects of response repetition from fully holistic perceptual priming where all features (brightness, size, and orientation) contribute to any effect of repetition.

A wealth of recent neurophysiological evidence, from single-cell studies (Bichot \& Schall, 2002), neuroimaging (Geng et al., 2006; Kristjánsson, Vuilleumier, Schwartz, Macaluso, \& Driver, 2007), neuropsychology (Kristjánsson, Vuilleumier, Malhotra, Husain, \& Driver, 2005; Saevarsson et al., 2008), transcranial magnetic stimulation (Campana, Cowey, \& Walsh, 2002, 2006; Muggleton, Juan, Cowey, Walsh, \& O’Breathnach, 2010; O’Shea, Muggleton, Cowey, \& Walsh, 2007), and lesion studies (Walsh, Le Mare, 
Blaimire, \& Cowey, 2000) indicates that priming in visual search reflects activity changes at a number of different neural loci: sites where objects may (at least to a considerable extent) be integrated, as well as areas that are involved in the processing of features (such as brightness, color, motion, or shape). Priming effects have been found as early as in the extrastriate cortex with fMRI (Kristjánsson et al., 2007).

Direct evidence for two-stage priming has come from Kristjánsson et al. (2008), who found that whether priming patterns reflect facilitated processing of integrated objects or features depends heavily on the type of stimulus that priming is tested for. Their observers searched for diamonds that contained two colors (on either side of their vertical midline) or a smaller diamond embedded within a larger one. These two types of stimuli have been shown in visual search studies (Wolfe, Friedman-Hill, \& Bilsky, 1994; Xu, 2002b) and studies of visual short-term memory (VSTM; Kristjánsson, 2006c; Vogel, Woodman, \& Luck, 2001; Xu, 2002a) to be processed differently by the visual system. The degree to which particular stimuli tended to be perceived as whole objects (when the smaller diamond was embedded within the larger) or separate features (the diamonds with different colors on either side of their vertical midline) determined strongly whether the effect from an object's repetition reflected what one might call object-based priming (which might overlap with processing stages proposed in episodic retrieval accounts) or featurebased priming. The results of Kristjánsson (2006b, 2009), where dissociable priming effects from repetition of different features were observed, also indicate that priming of whole objects is clearly not the whole story. Priming is thus likely to involve facilitation at several levels of the perceptual process (Kristjánsson \& Campana, 2010). Such dual- or multistage accounts have parallels in the literature on VSTM, where evidence suggests that VSTM consists of an early feature-based stage as well as a higher-level objectbased stage (Kristjánsson, 2006c; Olson \& Jiang, 2002; Wheeler \& Treisman, 2002).

Becker has also investigated the explanatory power of episodic retrieval accounts (Becker, 2008a; see also Becker, $2008 \mathrm{~b}$ ), finding that repetition of target features clearly modulated the accuracy and time course of the first saccade in a search sequence. These initial saccades were faster and more accurate when the same target was repeated than when it changed between trials, indicating that priming affects "the attention driving capacity of target and nontarget items on a trial-by-trial basis" (Becker, 2008a, p. 325). This suggests that priming affects search at an early stage of attentional guidance, before selection of the first search item. Becker and Horstmann (2009) argued, partly on the basis of these results of Becker (2008a), for a feature-weighting account of priming where priming is assumed to weight feature values similar to the target on the last trial more highly than others, a view broadly consistent with feature facilitation accounts. Analyses of eye movement patterns in tasks with low signal-to-noise ratios similar to the one introduced by Huang et al. (2004) would be of interest in future studies.

Some unanswered questions

There are some potentially interesting manipulations untested. An obvious manipulation would be to dissociate response repetition from feature repetition - for example, by introducing a present/absent version of Experiment 1 or a version with a less salient report feature. This might answer whether the size repetition by orientation repetition interaction reported by Huang et al. (2004) is due to response repetition or feature repetition. Another potentially informative manipulation concerns the guiding abilities of the relevant features. Comparing linearly inseparable stimulus sets with clearly separable ones would relate the results directly to a formal model of visual search (Wolfe, 2007; Wolfe et al., 1989) and well-documented abilities of visual features to guide attention (Wolfe \& Horowitz, 2004).

\section{Conclusions}

Our results indicate that the interaction between repetitions of different features used to argue for episodic retrieval accounts of priming is seen only when target signal strength against distractor noise is low. For less difficult searches with larger differences between targets and distractors, repetition effects from different features are independent of one another and, therefore, additive (as in Kristjánsson 2006b, 2009). This may reflect a dual-stage process similar to that in Lamy et al.'s (2010) account of priming in visual search or even a multistage process, as suggested by Kristjánsson and Campana (2010). A careful examination and quantification of target-distractor discriminability in visual search arrays may determine under which conditions priming reflects feature facilitation and when episodic retrieval.

Acknowledgements Jeremy Wolfe, Liqiang Huang, and two anonymous referees are thanked for very helpful comments on previous versions of the manuscript. The authors were supported by the Research Fund of the University of Iceland.

\section{References}

Becker, S. I. (2008a). The mechanism of priming: Episodic retrieval or priming of pop-out? Acta Psychologica, 127, 324-339. 
Becker, S. I. (2008b). The stage of priming: Are intertrial repetition effects attentional or decisional? Vision Research, 48, 664-684.

Becker, S. I. \& Horstmann, G. (2009). A feature weighting account of priming in conjunction search. Attention, Perception, \& Psychophysics, 71, 258-272.

Bichot, N. P., \& Schall, J. D. (2002). Priming in macaque frontal cortex during pop-out visual search: Feature-based facilitation and location-based inhibition of return. The Journal of Neuroscience, 22, 4675-4685.

Campana, G., Cowey, A., \& Walsh, V. (2002). Priming of motion direction and area V5/MT: A test of perceptual memory. Cerebral Cortex, 12, 663-669.

Campana, G., Cowey, A., \& Walsh, V. (2006). Visual area V5/MT remembers "what" but not "where." Cerebral Cortex, 16, 17661770.

Comtois, R. (2003). Vision Shell PPC [Software libraries]. Cambridge, MA: Author

Geng, J. J., Eger, E., Ruff, C., Kristjánsson, Á., Rothstein, P., \& Driver, J. (2006). On- line attentional selection from competing stimuli in opposite visual fields: Effects on human visual cortex and control processes. Journal of Neurophysiology, 96, 26012612.

Geyer, T., Müller, H. J., \& Krummenacher, J. (2006). Cross-trial priming in visual search for singleton conjunction targets: Role of repeated target and distractor features. Perception \& Psychophysics, 68, 736-749

Goolsby, B. A., \& Suzuki, S. (2001). Understanding priming of colorsingleton search: Roles of attention at encoding and "retrieval". Perception \& Psychophysics, 63, 929-944.

Hillstrom, A. P. (2000). Repetition effects in visual search. Perception \& Psychophysics, 62, 800-817.

Huang, L., Holcombe, A. O., \& Pashler, H. (2004). Repetition priming in visual search: Episodic retrieval, not feature priming. Memory \& Cognition, 32, 12-20.

Huang, L., \& Pashler, H. (2005). Expectation and repetition effects in searching for featural singletons in very brief displays. Perception \& Psychophysics, 67, 150-157.

Julesz, B. (1984). A brief outline of the texton theory of human vision. Trends in Neurosciences, 7, 41-45.

Kristjánsson, Á. (2006a). Rapid learning in attention shifts-A review. Visual Cognition, 13, 324-362.

Kristjánsson, Á. (2006b). Simultaneous priming along multiple dimensions in visual search task. Vision Research, 46, 25542570.

Kristjánsson, Á. (2006c). Surface assignment modulates objectformation for visual short-term memory. Perception, 35, 865881

Kristjánsson, Á. (2008). "I know what you did on the last trial"-A selective review of research on priming in visual search. Frontiers in Bioscience, 13, 1171-1181.

Kristjánsson, Á. (2009). Independent and additive repetition priming of motion direction and color in visual search. Psychological Research, 73, 158-166.

Kristjánsson, Á., \& Campana, G. (2010). Where perception meets memory: A review of repetition priming in visual search tasks. Attention, Perception, \& Psychophysics, 72, 5-18.

Kristjánsson, Á., \& Driver, J. (2008). Priming in visual search: Separating the effects of target repetition, distractor repetition and role-reversal. Vision Research, 48, 1217-1232.

Kristjánsson, Á., Ingvarsdóttir, Á., \& Teitsdóttir, U. D. (2008). Objectand feature-based priming in visual search. Psychonomic Bulletin \& Review, 15, 378-384.

Kristjánsson, Á., Vuilleumier, P., Malhotra, P., Husain, M., \& Driver, J. (2005). Priming of color and position during visual search in unilateral spatial neglect. Journal of Cognitive Neuroscience, 17, 859-873.

Kristjánsson, Á., Vuilleumier, P., Schwartz, S., Macaluso, E., \& Driver, J. (2007). Neural basis for priming of pop-out revealed with fMRI. Cerebral Cortex, 17, 1612-1624.

Kristjánsson, Á., Wang, D., \& Nakayama, K. (2002). The role of priming in conjunctive visual search. Cognition, 85, 37-52.

Lamy, D., Bar-Anan, Y., \& Egeth, H. E. (2008). The role of withindimension singleton priming in visual search. Journal of Experimental Psychology. Human Perception and Performance, 34, 268-285.

Lamy, D., Carmel, T., Egeth, H. E., \& Leber, A. B. (2006). Effects of search mode and intertrial priming on singleton search. Perception \& Psychophysics, 68, 919-932.

Lamy, D., Yashar, A., \& Ruderman, L. (2010). A dual-stage account of inter-trial priming effects. Vision Research, 50, 1396-1401.

Leonard, C. J., \& Egeth, H. E. (2008). Attentional guidance in singleton search: An examination of top-down, bottom-up, and intertrial factors. Visual Cognition, 8, 1078-1091.

Maljkovic, V., \& Nakayama, K. (1994). Priming of pop-out: I. Role of features. Memory \&Cognition, 22, 657-672.

Muggleton, N. G., Juan, C. H., Cowey, A., Walsh, V., \& O'Breathnach, U. (2010). Human frontal eye fields and target switching. Cortex, 46, 178-184.

Nakayama, K., Maljkovic, V., \& Kristjánsson, Á. (2004). Short term memory for the rapid deployment of visual attention. In M. S. Gazzaniga (Ed.), The cognitive neurosciences (3rd ed., pp. 397408). Cambridge: MIT Press.

O'Shea, J., Muggleton, N. G., Cowey, A., \& Walsh, V. (2007). Human frontal eye fields and spatial priming of pop-out. Journal of Cognitive Neuroscience, 19, 1140-1151.

Olivers, C. N. L., \& Meeter, M. (2006). On the dissociation between compound and present/absent tasks in visual search: Intertrial priming is ambiguity driven. Visual Cognition, 13, 1-28.

Olson, I. R., \& Jiang, Y. (2002). Is visual short-term memory object based? Rejection of the "strong-object" hypothesis. Perception \& Psychophysics, 64, 1055-1067.

Saevarsson, S., Jóelsdóttir, S., Hjaltason, H., \& Kristjánsson, Á. (2008). Repetition of distractor sets improves visual search performance in hemispatial neglect. Neuropsychologia, 45, $1161-1169$

Sigurdardottir, H. M., Kristjánsson, Á., \& Driver, J. (2008). Repetition streaks increase perceptual sensitivity in brief visual search displays. Visual Cognition, 16, 643-658.

Theeuwes, J. (1991). Cross-dimensional perceptual selectivity. Perception \& Psychophysics, 50, 184-193.

Treisman, A. M., \& Gelade, G. (1980). A feature-integration theory of attention. Cognitive Psychology, 12, 97-136.

Vogel, E. K., Woodman, G. F., \& Luck, S. J. (2001). Storage of features, conjunctions, and objects in visual working memory. Journal of Experimental Psychology. Human Perception and Performance, 27, 92-114.

Walsh, V., Le Mare, C., Blaimire, A., \& Cowey, A. (2000). Normal discrimination performance accompanied by priming deficits in monkeys with V4 or TEO lesions. Neuroreport, 11, 14591462.

Wang, D., Kristjánsson, Á., \& Nakayama, K. (2005). Efficient visual search without top-down or bottom-up guidance. Perception \& Psychophysics, 67, 239-253.

Wheeler, M. E., \& Treisman, A. M. (2002). Binding in short-term visual memory. Journal of Experimental Psychology. General, $131,48-64$.

Wolfe, J. M. (2007). Guided Search 4.0: Current progress with a model of visual search. In W. Gray (Ed.), Integrated models 
of cognitive systems (pp. 99-119). New York: Oxford University Press.

Wolfe, J. M., Butcher, S. J., Lee, C., \& Hyle, M. (2003). Changing your mind: On the contributions of top-down and bottom-up guidance in visual search for feature singletons. Journal of Experimental Psychology. Human Perception and Performance, 29, 483-502.

Wolfe, J. M., Cave, K. R., \& Franzel, S. L. (1989). Guided search: An alternative to the feature integration model for visual search. Journal of Experimental Psychology. Human Perception and Performance, 15, 419-433.
Wolfe, J. M., Friedman-Hill, S. R., \& Bilsky, A. B. (1994). Parallel processing of part-whole information in visual search tasks. Perception \& Psychophysics, 55, 537-550.

Wolfe, J. M., \& Horowitz, T. S. (2004). What attributes guide the deployment of visual attention and how do they do it? Nature Reviews. Neuroscience, 5, 495-501.

$\mathrm{Xu}, \mathrm{Y}$. (2002a). Encoding color and shape from different parts of an object in visual short-term memory. Perception \& Psychophysics, $64,1260-1280$.

$\mathrm{Xu}, \mathrm{Y} .(2002 \mathrm{~b})$. Feature integration across parts in visual search. Perception, 31, 1335-1347. 